\title{
Autoimmune regulator act in synergism with thymocyte adhesion in the control of IncRNAs in medullary thymic epithelial cells
}

\author{
Max Jordan Duarte ${ }^{1 \#}$, Romário S. Mascarenhas ${ }^{1 \#}$, Amanda Freire Assis ${ }^{1}$, Pedro \\ Paranhos Tanaka ${ }^{1}$, Cesar A. Speck-Hernandez ${ }^{2}$, Geraldo Aleixo Passos ${ }^{1,2,3,4^{*}}$
}

1 Molecular Immunogenetics Group, Department of Genetics, Ribeirão Preto Medical School, University of São Paulo (USP), Ribeirão Preto, SP, Brazil.

2 Program in Basic and Applied Immunology, Ribeirão Preto Medical School, University of São Paulo (USP), Ribeirão Preto, SP, Brazil.

3 Laboratory of Genetics and Molecular Biology, Department of Basic and Oral Biology, School of Dentistry of Ribeirão Preto, USP, Ribeirão Preto, SP, Brazil.

4 Center for Cell-Based Therapy in Dentistry, USP, Ribeirão Preto, SP, Brazil.

\#These authors contributed equally to this study and both are considered first authors.

* Corresponding author at: Molecular Immunogenetics Group, Department of Genetics, Ribeirão Preto Medical School, University of São Paulo (USP), 3900 Via Bandeirantes, Zipcode: 14049-900, Ribeirão Preto, SP, Brazil. E-mail: passos@usp.br 


\begin{abstract}
The autoimmune regulator (Aire) gene in medullary thymic epithelial cells (mTECs) encodes the AIRE protein, which interacts with its partners within the nucleus. This "Aire complex" induces stalled RNA Pol II on chromatin to proceed with transcription elongation of a large set of messenger RNAs and microRNAs. Considering that RNA Pol II also transcribes long noncoding RNAs (IncRNAs), we hypothesized that Aire might be implicated in the upstream control of this RNA species. To test this, we employed a loss-of-function approach in which Aire knockout mTECs were compared to Aire wildtype mTECs for IncRNA transcriptional profiling both in vitro and in vivo model systems. RNA sequencing enables the differential expression profiling of IncRNAs when these cells adhere in vitro to thymocytes or do not adhere to them as a way to test the effect of cell adhesion. Sets of IncRNAs that are unique and that are shared in vitro and in vivo were identified. Among these, we found the Aire-dependent IncRNAs as for example, Platr28, Ifi30, Morrbid, Malat1, and Xist. This finding represents the first evidence that Aire mediates the transcription of IncRNAs in mTECs. Microarray hybridizations enabled us to observe that temporal thymocyte adhesion modulates the expression levels of such IncRNAs as Morrbid, Xist, and Fbxl120 after $36 \mathrm{~h}$ of adhesion. This finding shows the existence of a synergistic mechanism involving a link between thymocyte adhesion, Aire, and IncRNAs in mTECs that might be important for immune self-representation.
\end{abstract}

Keywords: Aire gene, Crispr-Cas9, Cell adhesion, IncRNA, mTEC, mTEC-thymocyte adhesion

\title{
1. Introduction
}

The transcriptional control of gene expression in medullary thymic epithelial cells (mTECs) is a complex process, and according to recent findings, it involves two upstream controllers. One of these controllers is the autoimmune regulator (Aire) gene, which encodes the AIRE protein that functions as a transcriptional modulator, rather than as a classical transcription factor. Two features of the mode of action of AIRE classify it as a transcriptional modulator. First, AIRE associates with nuclear partner proteins, which interact with one another to form the AIRE complex. Next, the complex helps the stalled RNA Pol II to advance on chromatin and favors the transcription elongation phase in mTECs (Giraud et al., 2012), regulating a large set of downstream genes (Abramson et al., 2010; Passos et al., 2015, 2018; Perniola, 2018).

Forebrain embryonic zinc-finger-like protein 2 (Fezf2/FEZF2) is the second element that plays a role as a classical transcription factor that recognizes and interacts with promoter sequences on DNA (Takaba et al., 2015; Takaba and Takayanagi, 2017). The AIRE and FEZF2 proteins, although they display different modes of action, function in synergy, and both are under the control of the chromatin remodeler Chd 4 in promoting transcriptional control at the chromatin level (Tomofuji et al., 2020). 
In addition, the Aire gene is also involved in posttranscriptional control in mTECs by regulating the expression of miRNAs, which, in turn, negatively regulate downstream mRNAs (Macedo et al., 2013; Ucar et al., 2015; Oliveira et al., 2016).

Notable advances have been made in understanding and characterizing the mammalian cell transcriptome. As previously discussed (Assis et al., 2014), in functional terms, only a portion of the mammalian genome encodes RNAs, from which only a small fraction encode proteins; therefore, most RNAs are nonprotein-coding. Among the various species of mammalian RNAs described to date, a class of noncoding RNAs measuring more than 200 nucleotides, known as long noncoding RNAs (IncRNAs), have been attracting attention recently (Yao et al., 2019; Gil and Ulitsky, 2020; Bhatti et al., 2021a; 2021b; Song et al., 2021).

LncRNAs play roles as regulatory molecules that interact with other molecules, such as stretches of genomic DNA, proteins, miRNAs, or mRNAs (Zhang et al., 2018). The IncRNAs are implicated in such biological processes as normal eukaryotic development (Jarroux, Morillon and Pinskaya, 2017), cell differentiation and pluripotency (Chen et al., 2017; Yan et al., 2017), cancer biology (Bhan et al., 2017), the inflammatory response (Mathy and Chen, 2017) and autoimmunity (Zemmour, 2017). Among the subcategories of IncRNAs, intergenic IncRNAs, or simply lincRNAs, play a variety of roles, ranging from chromatin remodeling and RNA stabilization to the regulation of transcription (Ransohoff, Wei and Khavari, 2018).

However, the roles played by IncRNAs in the normal functioning of the thymus gland or in isolated thymic epithelial cells (TECS) have not been fully elucidated, and to the best of our knowledge, the few published results to date concern the effect of beta-estradiol on IncRNA expression in mTECs (Wei et al., 2018), thymic tumors (Su et al., 2020), effect of Zika virus on thymus (Messias et al., 2020) and thymic involution in birds (Li et al., 2021).

In this study, we hypothesized that Aire plays a role in the upstream control of IncRNAs in mTECs. To test this hypothesis, we used large-scale gene expression methods, such as next-generation RNA sequencing (RNA-Seq) and microarray hybridizations, to profile the IncRNA transcriptome of Aire wild type or Crispr-Cas9generated Aire knockout (KO) mTECs in an in vitro model system.

Since the transcriptional activity of mTECs and their functionality is guaranteed through cell adhesion (Pezzi et al., 2016; Speck-Hernandez et al., 2018; Cotrim-Sousa et al., 2019; Irla, 2019), we also monitored the expression profiles of IncRNAs during the in vitro temporal adhesion to thymocytes.

Moreover, to evaluate the extension of the Aire effect on IncRNAs in vivo, we analyzed data of mTECs isolated of Aire wild type or of Aire KO mice.

\section{Materials and Methods}

\subsection{Mice and separation of thymocytes}

We employed C57BL/6 mice from the Jackson Laboratory (Bar Harbor, ME, USA) that were housed and mated at the Central Animal Facility, University of São 
Paulo, Campus of Ribeirão Preto, SP, Brazil, in temperature-controlled (approximately $22^{\circ} \mathrm{C}$ ) and specific-pathogen-free conditions in $0.45-\mu \mathrm{m}$ air-filtered ventilated racks under 12-h dark/light cycles and receiving water and food ad libitum.

For the surgical removal of the thymus and further separation of thymocytes, we employed four- to five-week-old females weighing 18-22 g using a previously described protocol (Donate et al., 2013). Before surgery, each animal was individually placed into an acrylic cage connected through a silicon pipe to a steel cylinder containing pressurized $\mathrm{CO}_{2}$, and the animal was euthanized by gas inhalation.

Thymi were removed from the animals, dissected and fragmented in RPMI 1640 medium. The thymocytes were collected by 2-3 passages of the thymic fragments through a $10-\mu \mathrm{m}$ mesh nylon membrane (Sefar Inc., Depew, NY, USA) and centrifuged. The pelleted thymocytes were resuspended in phosphate-buffered saline (PBS) pH 7.4.

Fluorescence-activated cell sorting (FACS) analysis was performed using a BD FACSCalibur (BD Biosciences) flow cytometer with a phycoerythrin (PE)-labeled antiCD3 antibody (Biolegend CNS, Inc. San Diego, CA, USA) and indicated that this procedure yielded a thymocyte population with a purity of $\geq 90 \%$. These cells were utilized for further cell adhesion assays. All experiments with mice had been previously analyzed and approved by the Animal Research Ethics Committee, University of São Paulo, Campus of Ribeirão Preto, Brazil (approval number 003/2017-1).

\subsection{Aire wild-type and knockout medullary thymic epithelial cell lines (mTECs)}

For the in vitro experiments, we employed the murine ( $M$. musculus) Aire wildtype (WT) mTEC line (EpCAM ${ }^{+}$, Ly51$^{-}, \mathrm{UEA}^{+}$) of $\mathrm{mTEC} 3.10$ cells, as previously described (Nihei et al., 2003; Hirokawa et al., 1986). Besides it features the EpCAM ${ }^{+}$, Ly51; , UEA1 ${ }^{+}$phenotype, these cells also express Aire (Oliveira et al., 2013; 2016), Ccl21 and Sap1 genes (this work from the RNA-seq or microarray data) and the Aire protein (Supplemental material Figure 1). According to recent $\mathrm{mTEC}$ characterizations (Ribeiro et al., 2019), the MTEC 3.10 cell line is compatible with the ITEC $^{\text {low }}$ differentiation stage.

Moreover, we previously have shown that the Aire WT mTEC 3.10 line express the Aire protein, which is localized in the nucleus of mTECs as shown through immunofluorescence (Pezzi et al., 2016; Speck-Hernandez et al., 2018).

In addition, we used a Crispr-Cas9-generated Aire knockout (KO), which was derived from the wild type mTEC 3.10 cell line and is known as the MTEC 3.10 E6 clone.

The MTEC 3.10E6 clone was characterized as a KO compound heterozygous. This cell line is a carrier of indel mutations targeting exon 3 of the Aire gene and affecting both alleles, which feature different mutations. In Aire allele 1 , there are two types of mutations: a $T>G$ substitution (at position 351 of the encoded mRNA) followed by a nine-bp deletion (GCTGGTCCC, at positions 352-360 of the encoded mRNA) that results in the transcription of a 1,647-nucleotide Aire mRNA. In Aire allele 2 , a single $\mathrm{G}$ deletion corresponds to position 352 of the encoded mRNA (resulting in a 
1,655-nucleotide Aire mRNA). The $T>G$ substitution in allele 1 causes the encoded AIRE protein to have a silent mutation (L118L).

In contrast, the nine-base-pair deletion caused a frameshift with a deletion of three amino acid residues (A119_P121del) and, consequently, a shorter and nonfunctional 548-amino-acid AIRE protein (Speck-Hernandez et al., 2018). FASTA sequences of Aire exon 3 are available at GenBank NCBI (https://www.ncbi.nlm.nih.gov/nucleotide/) under accession numbers MG493266 for Aire mutant allele 1 and MG493265 for Aire mutant allele 2. WT or KO mTECs were employed for further cell adhesion assays.

The Aire KO mTEC 3.10E6 mutant cell line does not localize Aire protein in the nucleus as previously shown through immunofluorescence (Speck-Hernandez et al., 2018).

For the in vivo model system analysis, we retrieve previously generated RNASeq data from mTECS ( $m$ TEC $C^{\text {high }}$ or mTEC $^{\text {low }}$ ) isolated from Aire wild type or Aire KO mouse fresh thymi (St Pierre et al., 2015) whose raw data is available at Gene Expression Omnibus (GEO) available at (https://www.ncbi.nlm.nih.gov/geo/) under the accession number GSE65617.

\section{3 mTEC-thymocyte cell adhesion assay}

To evaluate the effect of deleterious Aire mutations (KO) in mTECs on thymocyte adhesion, we utilized a previously established protocol (Savino et al., 2004, 2010; Oliveira et al., 2016) with modifications, as described (Pezzi et al., 2016): specifically, the mTEC cells were cultured in RPMI 1640 medium containing $10 \%$ fetal bovine serum and antibiotics at $37^{\circ} \mathrm{C}$ in a $5 \% \mathrm{CO}_{2}$ atmosphere. Semiconfluent cells were detached from their culture flasks by conventional trypsin/EDTA treatment, washed once with PBS at room temperature, resuspended in RPMI 1640 medium, and seeded in new culture flasks $\left(2 \times 10^{6}\right.$ cells per $75 \mathrm{~cm}^{2}$ Corning ${ }^{\circledR}$ cell culture flasks). For this study, WT mTEC 3.10 cells or Aire KO mTEC 3.10E6 cells were cocultured with thymocytes, as described below.

Thymocytes were added to WT MTEC 3.10 or Aire KO mTEC 3.10 E6 cultures at a ratio of 50:1 (thymocyte:mTEC) and cocultured in RPMI medium containing $10 \%$ fetal bovine serum and antibiotics at $37^{\circ} \mathrm{C}$ in a $5 \% \mathrm{CO}_{2}$ atmosphere. In a typical adhesion experiment, the cell input was adjusted as follows: $5 \times 10^{6}$ thymocytes and $1 \times 10^{4}$ mTECs. This gives a 50: 1 ratio (thymocytes: mTECs). Next, the nonadherent thymocytes were carefully removed from cultures by washing with PBS at $37^{\circ} \mathrm{C}$ and discarded. The culture flasks were washed more vigorously with PBS at $4^{\circ} \mathrm{C}$ to remove the adherent thymocytes, which were kept for counting and phenotyping. Singlepositive (SP) $\mathrm{CD}^{+}$or $\mathrm{CD}^{+}$cells were stained with an APC-Cy7-labeled anti-CD4 and FITC-labeled anti-CD8 antibody (Biolegend), respectively, and quantified using a BD LRSFortessa flow cytometer (BD Biosciences).

Next, mTEC cells were detached from their culture flasks by conventional trypsin/EDTA treatment and resuspended in PBS for counting. Cell counts for either thymocytes or mTECs were performed on a Cellometer Auto T4 Cell Viability Counter (Nexcelon Bioscience, Lawrence, MA, USA). Cells were recovered from cocultures after 
12 or $36 \mathrm{~h}$, which correspond to two incubation time points that give linear response in terms of thymocyte adhesion (data not shown).

Experiments were performed in triplicate (i.e., three co-cultures with Aire WT mTEC 3.10 and three cocultures with Aire KO mTEC 3.10E6) and repeated at least three times, with similar results being obtained. The adhesion index (Al) was calculated as follows: $\mathrm{Al}=$ number of adhered thymocytes/number of mTEC cells. Statistical analysis was performed by Student's $t$-test using the GraphPad Prism 6.0 platform. P-values < 0.05 were considered to be significant.

\subsection{Total RNA preparation and reverse transcription real-time quantitative PCR (RT- qPCR)}

We followed previously described protocols (Pezzi et al., 2016; Cotrim-Sousa et al., 2019). Briefly, total RNA was prepared using the mirVana ${ }^{\circledR}$ kit (Ambion, Austin, TX, USA) according to the manufacturer's instructions. Through UV spectrophotometry, RNA preparations were confirmed to be free of proteins and phenol. RNA degradation was assessed by microfluidic electrophoresis using Agilent RNA 6000 nanochips and an Agilent 2100 Bioanalyzer (Agilent Technologies, Santa Clara, CA, USA). Only RNA samples that were free of proteins and phenol and had RNA integrity numbers (RINs) $\geq$ 9.0 were employed for CDNA synthesis with SuperScript reverse transcriptase enzyme according to the manufacturer's instructions (Invitrogen Corporation, Carlsbad, CA, USA).

RT-qPCR was employed to confirm the expression levels of Fendrr, Peg13, Fam219aos, Platr28 and Neat1 IncRNAs. The expression level of each IncRNA was normalized to the housekeeping mRNA Hprt; these gene is commonly used as reference. The Primer3 web tool (http://frodo.wi.mit.edu/primer3) was utilized to select pairs of oligonucleotide primers spanning an intron/exon junction with an optimal melting temperature of $60^{\circ} \mathrm{C}$.

The cDNA sequences of IncRNAs were retrieved from the NCBI GenBank Database (http://ncbi.nlm.nih.gov/nuccore?itool=toolbar). The forward (F) and reverse (R) sequences (presented in the 5' to 3' orientation) were the following: mRNA Hprt (NM_013556.2) CCCCAAAATGGTTAAGGTT and CAAGGGCATATCCAACAACA, InCRNA Fendrr (NR_130109) CTCTCCAGTTCCCACCACC and TGGTCTGGTTCTGGTCACTC, InCRNA Peg13 (NR_002864)TGTGACCACGAACCGAAGAG and TCGTCTACATAGCACCAGCG, InCRNA Fam219aos (NR_045726) AGGCAGAGGTTCTGATTCACAG and GTGAAAAACTGACCCCCTGGA, InCRNA Platr28 $\quad$ (NM_001018010) TTGGAGCACAAAATGGGACCT and GGGCCAAGCTCAGGTCAAC, IncRNA Neat1 (NR_003513) CTGCACTGTAGATCGGGACC and TCCCCAACACCCACAAGTTT.

Transcription was quantified using a StepOne Real-Time PCR System (Applied Biosystems, USA). The analyses were performed using the cycle threshold ( $\mathrm{Ct}$ ) method, which enables quantitative analysis of the expression of a factor using the formula 2$\mathrm{D} \Delta \mathrm{C}^{\wedge} \mathrm{t}$, in which $\Delta \mathrm{Ct}=\mathrm{Ct}$ target cDNA sequence $-\mathrm{Ct}$ of the housekeeping mRNA Hprt, and $\Delta \Delta \mathrm{Ct}=\Delta \mathrm{Ct}$ sample $-\Delta \mathrm{Ct}$. 


\subsection{Western blot of AIRE protein}

Western blotting (WB) of AIRE protein expressed in Aire wild type mTEC 3.10 or Aire KO mTEC 3.10E6 cell lines, was performed according a previously published protocol optimized in our laboratory (Pezzi et al., 2016). Briefly, we use a primary antiAIRE goat polyclonal antibody (Anti-AIRE D17 IgG antibody, Santa Cruz Biotechnology Inc., Dallas, TX, USA) and a horseradish peroxidase (HRP)-conjugated donkey anti-goat IgG as secondary antibody (Merck Millipore, Darmstadt, Germany).

The WB polyvinylidene fluoride (PVDF) membrane (BioRad, Hercules, CA, USA) was developed using a peroxidase substrate for chemiluminescence (LuminataForte ${ }^{\mathrm{TM}}$, Merck Millipore) in an ImageQuant ${ }^{\text {TM }}$ LAS 500 apparatus (GE Life Sciences, Piscataway, NJ, USA).

Protein bands were visualized using the ImageQuant TM GE Life Sciences (Piscataway, NJ, USA) and quantitatively analyzed with ImageJ software (version 1.49). AIRE protein was normalized to GAPDH protein, which is routinely used as a housekeeping. The same membrane that was used to detect AIRE protein was washes and reused in an incubation with an anti-GAPDH rabbit polyclonal primary antibody (Cell Signaling Technology, Beverly, MA, USA) following incubation with peroxidase conjugated anti-rabbit antibody.

The membrane was developed as described above. Statistical comparison of protein bands intensity between in Aire wild type mTEC 3.10 and Aire KO mTEC 3.10E6 cell lines was performed using Student's t-test within the GraphPadPrism 6.0 platform (http://www.graphpad.com/prism/Prism.htm).

\subsection{RNA sequencing (RNA-Seq)}

This analysis was based on the protocol as previously described (SpeckHernandez et al., 2018). Briefly, paired-end ( $2 \times 100$ bp) sequencing was performed using an Illumina HiSeq 2500 sequencer (Illumina, San Diego, CA, USA) installed at LacTad Laboratory Facility, Campinas State University, Campinas, SP, Brazil. To this end, we employed a TruSeq Stranded Total RNA Library Prep Kit (Illumina).

Data were analyzed following this process: the quality of raw FASTQ sequences

was first analyzed through a FASTQC program (https://www.bioinformatics.babraham.ac.uk/projects/fastqc/). FASTQC sequences were mapped to the Mus musculus reference genome (mm10) using the STAR 2.5 Spliced Aligner program (https://github.com/alexdobin/STAR), which output a BAM file containing the sequences and their genomic references, as well as a GFT file with gene annotations employed for further determinations of the number of reads per transcript through the HTSeq Count program (https://htseq.readthedocs.io/en/master/).

The edger package (https://bioconductor.org/packages/release/bioc/html/edgeR.html) was employed to analyze the differentially expressed (DE) IncRNAs in Aire KO mTEC 3.10E6 compared to Aire WT mTEC 3.10 cells before and after thymocyte adhesion. We considered DE 
IncRNAs with fold changes $\geq 2.0$ and adjusted P-values (FDR) $\leq 0.05$. The RNA-Seq data obtained in this work are available on GEO under the accession number GSE91015.

For the analysis of DE IncRNAs expressed in vivo by mTECs isolated of Aire wild type or Aire KO fresh thymi, we used the same pipeline as above described.

\subsection{Microarray hybridization and data analysis}

We utilized the protocol established by our group and previously published (Donate et al., 2013; Oliveira et al., 2013; Fornari et al., 2015). Briefly, the gene expression changes were evaluated using the Agilent one-color (Cy3 fluorochrome) microarray-based gene expression platform according to the manufacturer's instructions. To hybridize mRNAs or IncRNAs to whole-genome $8 \times 60 \mathrm{~K} 60$-mer oligonucleotide arrays (Agilent Technologies, Palo Alto, CA, USA), $200 \mathrm{ng}$ of total RNA was labeled with the one-color Quick Amp labeling kit (Agilent Technologies).

Samples of complementary RNA (CRNA) were hybridized for $18 \mathrm{~h}$ at $42^{\circ} \mathrm{C}$ in a rotator oven and washed. The array slides were scanned using a DNA microarray scanner (Agilent Technologies), and the hybridization signal was extracted using Agilent Feature Extraction software (version 10.5). Gene expression profiles from two or three independent preparations of control (mTEC 3.10 cells) and Aire KO (mTEC $3.10 \mathrm{E} 6$ cells) before or after thymocyte adhesion were analyzed by comparing the microarray hybridizations of the respective samples.

A complete file, including all of the oligo sequences present in the microarray representing mRNAs or IncRNAs employed in this study and the experimental conditions, is available online in the ArrayExpress public database (https://www.ebi.ac.uk/arrayexpress/) under accession number E-MTAB-6541.

The quantitative microarray data were normalized to the 75th quantile and analyzed using the $\mathrm{R}$ platform (https://www.r-project.org/) (version 3.10). Computational tools contained in the arrayQualityMetrics (https://www.bioconductor.org/packages/release/bioc/html/arrayQualityMetrics.html ) (Kauffmann, Gentleman and Huber, 2009), and Agi 4 x 44 PreProcess (López-Romero et

al., 2010),

(http://www.bioconductor.org/packages//2.11/bioc/manuals/Agi4x44PreProcess/man /Agi4x44PreProcess.pdf) were utilized for the preprocessing of the data. Bioconductor packages contain algorithms for assessing the quality of the data, background correction, and normalization. After analyzing the DE mRNAs or IncRNAs, functions contained in the limma package (https://bioconductor.org/packages/release/bioc/html/limma.html) (Wettenhall and Smyth, 2004), were employed.

This study considered only those mRNAs or IncRNAs with adjusted P-values $\leq 0.05$, controlled false discovery rates (FDR) by the Benjamini-Hochberg method, and fold changes $\geq 1.5$ to be DE. The DE IncRNAs were hierarchically clustered. Heat maps were constructed using Euclidean distance and the complete linkage method to evaluate controls and Aire KO mTECs' expression profiling before and after thymocyte adhesion. As described above for RNA-seq data, we utilized the IncTAR (http://www.cuilab.cn/Inctar) and CPC2 (http://cpc2.cbi.pku.edu.cn/) for the functional enrichment of IncRNAs. 


\subsection{Identification and nomenclature of differentially expressed IncRNAs}

The identification of IncRNAs was performed according to their annotations in the UCSC Genome Browser (https://genome.ucsc.edu/) and Agilent's annotation regarding the microarray slides.

\subsection{Identification and genomic positioning of protein-encoding genes near IncRNAs}

The $\mathrm{R}$ platform (https://www.r-project.org/) and biomaRt package (https://www.ensembl.org/biomart/martview/) were utilized to access databases, including Ensembl (https://www.ensembl.org/), to search in the genome the proteincoding genes closest to the IncRNAs.

\subsection{Evaluation of the coding potential of IncRNAs}

The coding potential of differentially expressed IncRNAs was analyzed using CPC2 software (http://cpc2.cbi.pku.edu.cn/), a calculator that assesses the coding potential of a given IncRNA using an alignment-free technique. This software employs a logistic regression model based on four characteristics: 1) the size of the open reading frame (ORF, or open reading matrix), since long ORFs are rarely observed in noncoding sequences; 2) the ORF coverage, that is, the ratio between ORF and transcript lengths; 3) the Fickett TESTCODE score, which reflects the possible combinatorial effect of the nucleotide composition and the tendency to use a particular codon; and 4) the hexamer score, which determines the relative degree of bias in the use of a hexamer in a given sequence (Kong et al., 2007; Kang et al., 2017).

The values of this score vary from 0 to 1 . Values close to 0 indicate lower coding potential, and as they approach 1 , they indicate more significant coding potential based on the cutoff of 0.5 (Kang et al., 2017). We analyzed the coding potential only of the differentially expressed IncRNAs cataloged at Ensembl (https://www.ensembl.org/index.html).

\section{Results}

\section{1 mTEC-thymocyte adhesion is influenced by Aire}

For the cell adhesion assay, we used murine ( $M$. musculus) mTECs (Aire WT mTEC 3.10 or Aire KO mTEC 3.10E6 cell lines). The mTEC-thymocyte coculture for 12 or $36 \mathrm{~h}$ showed a significant reduction in the number of adhered thymocytes on Aire KO mTEC 3.10E6 cells as demonstrated throughout a conventional cell adhesion assay and whose mTECs and thymocytes were visualized by light microscopy. In order to calculate the adhesion index (Al) both cell types from cocultures were individually counted. Since in physiological conditions and within the thymus, single positive (SP) thymocytes adhere to mTECs, we use flow cytometry analysis to show that in vitro an equal proportion between SP $\mathrm{CD} 4^{+}$and $\mathrm{CD} 8^{+}$thymocytes were adhered to this cell 
bioRxiv preprint doi: https://doi.org/10.1101/2021.07.14.452339; this version posted July 14,2021 . The copyright holder for this preprint (which was not certified by peer review) is the author/funder, who has granted bioRxiv a license to display the preprint in perpetuity. It is made available under aCC-BY-NC-ND 4.0 International license.

type. Thymocyte adhesion was lowered with Aire KO mTEC $3.10 \mathrm{E} 6$ and was not preferred for one of the SP subtypes (Figure 1).

A

WT
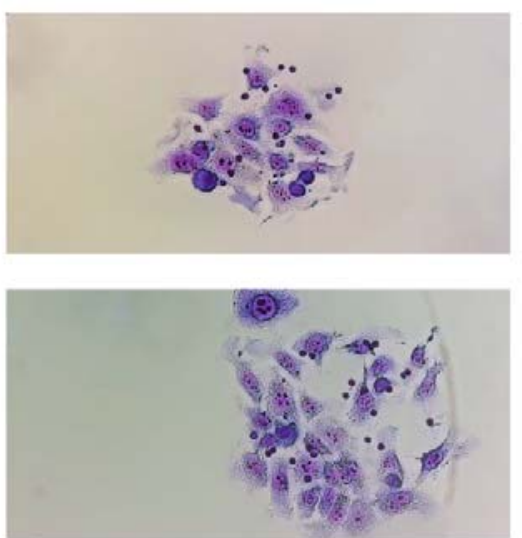

KO
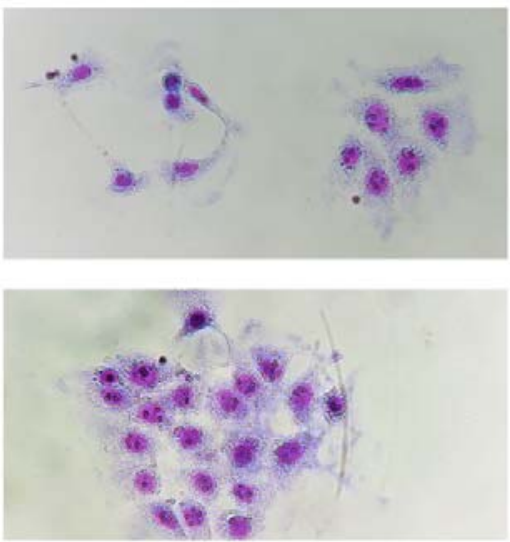

B

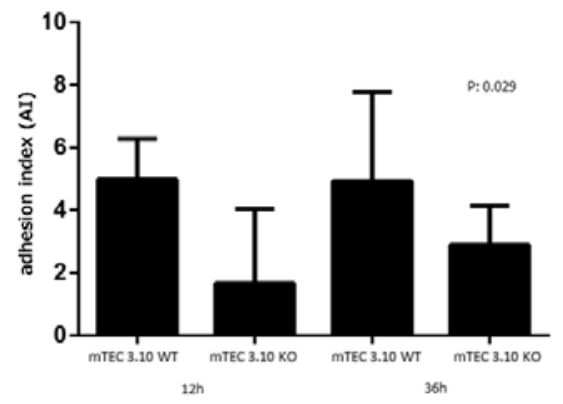


C

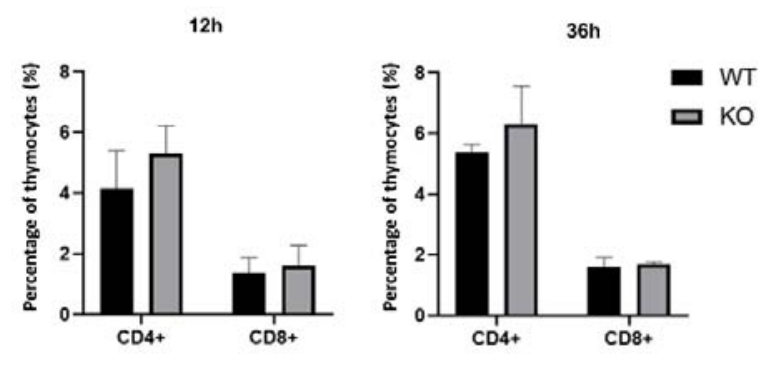

D

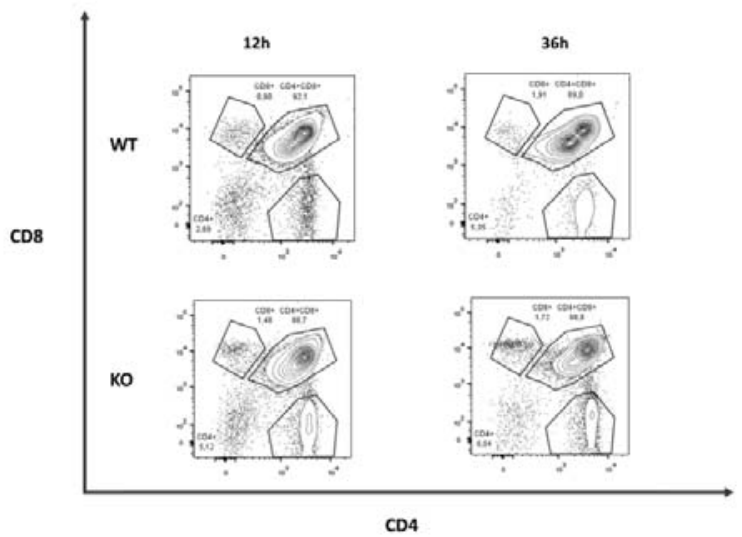

Figure 1 The mTEC-thymocyte adhesion was comparing Aire wild-type (WT) mTEC 3.10 with Aire KO mTEC 3.10E6. (A) Photomicrography of fresh mTEC-thymocyte co-cultures after $12 \mathrm{~h}$ or $36 \mathrm{~h}$ adhesion. Conventional light microscopy, Giemsa staining, $20 \mathrm{x}$ magnification. (B) Adhesion indexes after 12 or $36 \mathrm{~h}$ mTEC-thymocyte co-cultures. (C) Quantification of CD4 and CD8 cell surface markers of adhered or non-adhered thymocytes to mTECs. (D) Flow cytometry of CD4 single-positive (SP) thymocytes and CD8 SP adhered or nonadhered thymocytes to mTECs and CD4/CD8 double-positive thymocytes adhered to Aire wild type (WT) mTEC 3.10. 


\subsection{Differential expression of IncRNAs is influenced by Aire and thymocyte adhesion}

Initially we use RNA-Seq to identify the differentially expressed (DE) IncRNAs between mTEC samples. The 136 DE IncRNAs as observed in the unsupervised hierarchical clustering heat map of Figure 2, were considered Aire-dependent, of which 72 were upregulated, and 64 were downregulated when comparing Aire wild type (WT) vs Aire knockout (KO) mTECs subjected, or not, to thymocyte adhesion. This result demonstrate the influence of Aire and thymocyte adhesion on IncRNAs in mTEC cells. The top ten DE IncRNAs are presented in Supplemental material Table 1 and, Table 1.1.

A

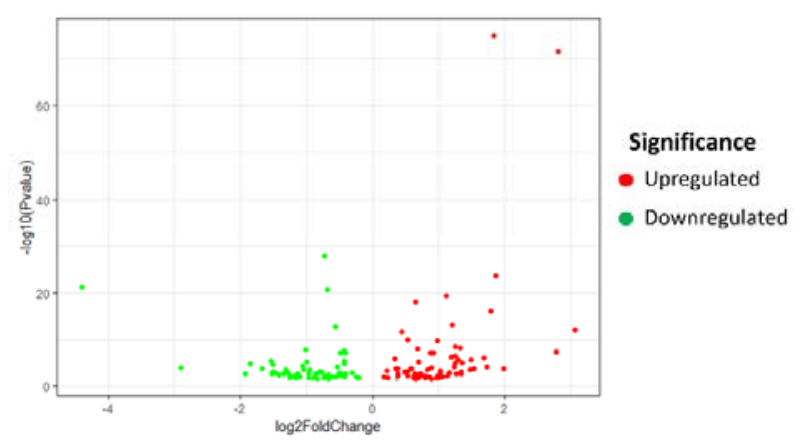

B

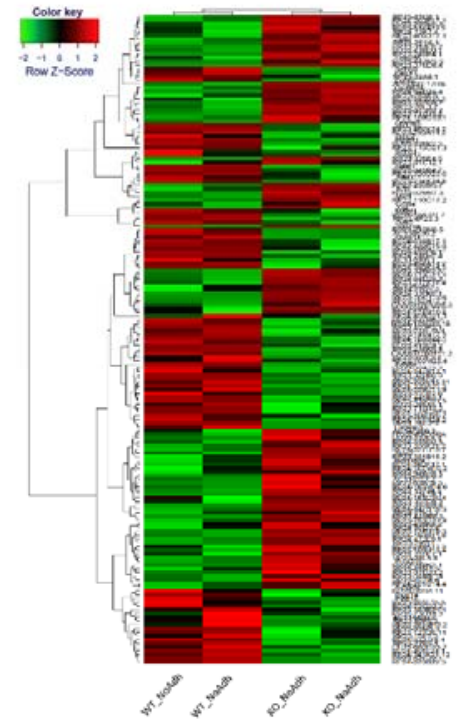

Figure 2 Differentially expressed IncRNAs were considered Aire-dependent, of which 72 were upregulated, and 64 were downregulated when comparing Aire wild type (WT) vs Aire knockout (KO) mTECS subjected, or not, to thymocyte adhesion. Total RNA samples were extracted from WT or Aire KO mTECs and sequenced through Illumina protocol (RNASeq). (A) Volcano plot showing the differentially expressed IncRNAs in Aire KO mTEC 3.10E6. Red dots represent the upregulated and green dots, the downregulated IncRNAs, fold-change $\geq 2.0$. (B) Heat map showing differentially expressed IncRNAs in Aire KO mTEC 3.10E6. Red means the upregulated and green the downregulated IncRNAs. Row $Z$ scores, fold-change $\geq 2.0$ normalize heat-map colors. 


\subsubsection{Effect of temporal thymocyte adhesion on mTEC IncRNA expression}

In order to evaluate whether temporal thymocyte adhesion may cause differences in the expression profile of IncRNAs, we analyzed their expression throughout microarray hybridizations varying the coculture time. The Venn diagram depicted in Figure 3, shows 45 identified DE IncRNAs when comparing Aire WT mTEC 3.10 vs. Aire KO mTEC $3.10 E 6$ cells before thymocyte adhesion. Of these, eight IncRNAs were upregulated, and 37 were downregulated. After $12 \mathrm{~h}$ of thymocyte adhesion, 53 DE InCRNAs (16 up- and 37 downregulated) were observed, and after $36 \mathrm{~h}$ of adhesion, 138 DE IncRNAs (31 up- and 107 downregulated) were identified (Figure 3).

Figure 4 shows the hierarchical clustering of the transcriptional expression of IncRNAs, comparing Aire WT mTEC 3.10 with Aire KO mTEC 3.10 E6 cells before and after temporal thymocyte adhesion, with adjusted P-values $\leq 0.05$ (BenjaminiHochberg FDR) and fold-changes $\geq 1.5$ serving as the cutoff, as shown in the heat map.

A

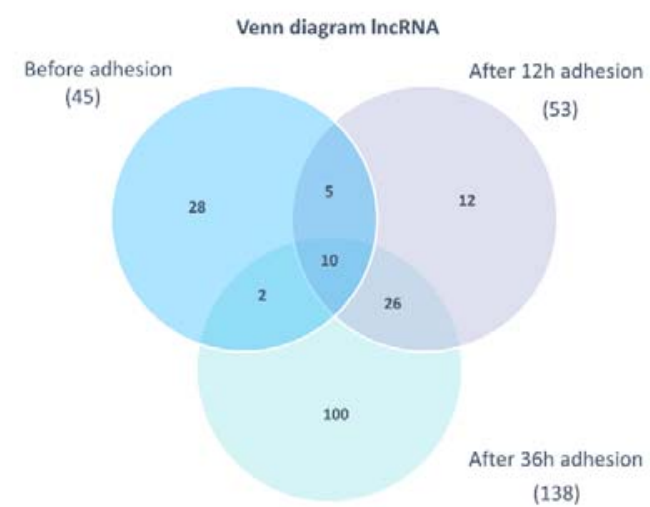

B

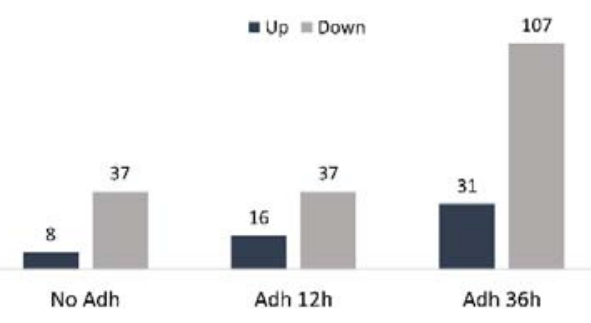

Figure 3 (A) Venn diagram of the differently expressed IncRNAs comparing Aire wild-type (WT) mTEC 3.10 with Aire KO mTEC 3.10E6 before and after thymocyte adhesion. (B) Summary of differently expressed IncRNAs. Total RNA samples were extracted from WT or Aire KO mTECs subjected or not to thymocyte adhesion during 12 or $36 \mathrm{~h}$, labeled and hybridized to Agilent microarrays. 


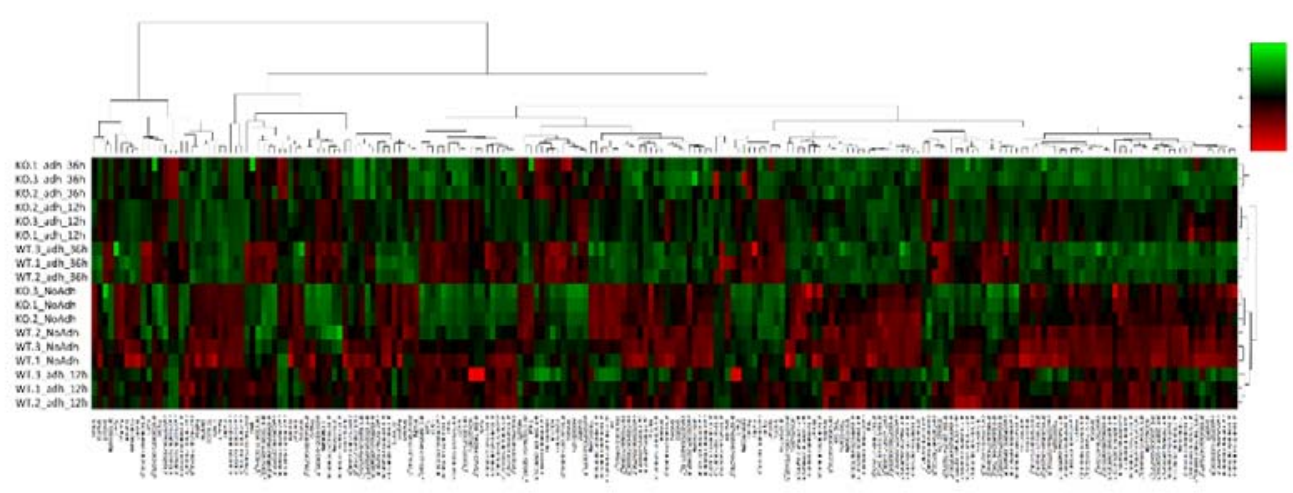

Figure 4 Heat map plot of differentially expressed IncRNAs comparing Aire wild-type (WT) mTEC 3.10 with Aire KO mTEC 3.10E6 before and after thymocyte adhesion. Total RNA samples were extracted from WT or Aire KO mTECs subjected or not to thymocyte adhesion during 12 or $36 \mathrm{~h}$, labeled and hybridized to Agilent microarrays. Unsupervised dendrograms and heatmap were constructed using the $\mathrm{R}$ platform. Red = upregulated mRNAs, green = downregulated mRNAs and black = unmodulated mRNAs (Fold-change $\geq 2.0$, false discovery rate $F D R \leq 0.05$, Pearson correlation metrics, 75 percentile).

\subsection{Extent of the effect of Aire in the IncRNA expression in vitro and in vivo}

Then we asked if Aire could influence the expression of mTEC IncRNAs both in vitro and in vivo. For this, we compared the expression data of IncRNAs collected in this study that uses the in vitro mTEC-thymocyte adhesion with the expression of IncRNAs from mTECs separated from fresh mouse thymus. The Venn diagram depicted in Figure 5 shows that either in vivo or in vitro the absence of Aire influences the number of DE IncRNAs. As the expression data from Aire wild type mTECs were used as a reference, the Venn diagram was constructed with data from Aire KO mTECs in which appear the shared and the exclusive IncRNAs according to the sample analyzed, i.e., Aire KO mTEC $^{\text {low }}$ vs Aire KO mTEC ${ }^{\text {high }}$ isolated from in vivo fresh thymus (diagram left side) or Aire KO mTEC without thymocyte adhesion vs Aire KO mTEC under in vitro thymocyte adhesion (diagram right side). 


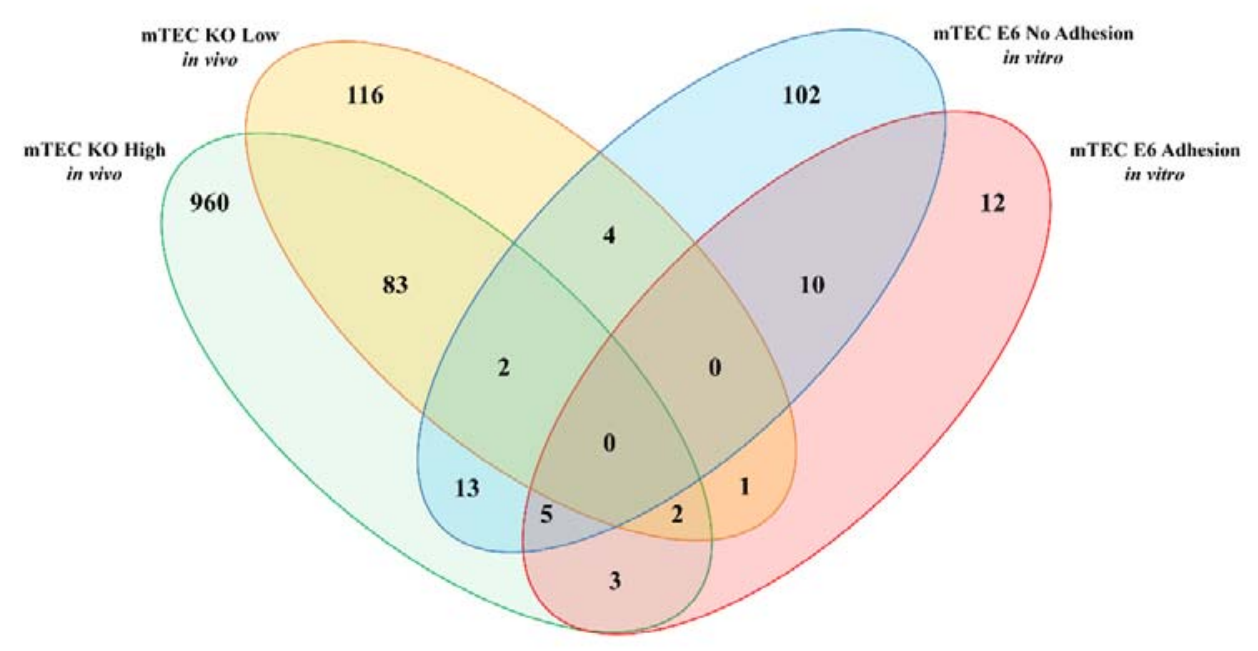

Figure 5 Venn diagram showing the exclusive and shared differentially expressed IncRNAs in Aire KO mTECs. In vivo Aire KO mTEC ${ }^{\text {low }}$ / Aire KO mTEC ${ }^{\text {high }}$ (left side) and in vitro Aire KO mTEC with no thymocyte adhesion / Aire KO mTEC under thymocyte adhesion (right side). The RNASeq data from the in vivo mTECs were retrieved from St Pierre et al., (2015) and from the in vitro mTECs, this study.

\subsection{Positioning of protein coding genes near IncRNA genes}

Regarding the relative position in the genome of protein-encoding genes to IncRNAs, we located a wide range both upstream and downstream considering the different chromosomes. For example, while the Neat1 IncRNA is found 29,796 bp upstream of the Frmd8 protein-encoding gene, this IncRNA is 74,059 bp downstream of the Scyl1x gene. Also, the IncRNA Meg3 is 59,329 bp upstream of the Rtl1 gene and 88,178 bp downstream of the Dlk1 gene (Supplemental material Table 2, Table 2.1 and, Table 2.2).

\subsection{Coding potential of IncRNAs}

Among the RNAs that are differentially expressed (DE) in mTECs before their adhesion with thymocytes, we found 13 that were unlikely to encode a protein and were therefore classified as IncRNAs (Supplemental material Table 3). After $12 \mathrm{~h}$ of thymocyte adhesion, we listed 27 differentially expressed RNAs, 26 of which were classified as IncRNAs along with an exception of the DNAH10-202. Although DNAH10202 falls into this classification, it presents coding potential (Supplemental material Table 3.1). Next, after $36 \mathrm{~h}$ of thymocyte adhesion, we listed 56 differentially expressed IncRNAs, among which the following exceptions presented coding potential (1700018B24Rik, DNAH10-202 shared with 12-h adhesion, E230016K23Rik, Gm10069, Gm14636, Gm16062, Gm20324, and Gm50039) (Supplemental material Table 3.2). 


\subsection{Validation of the transcriptional expression levels by RT-qPCR}

To confirm the modulation of selected IncRNAs we evaluated the modulation of Fendrr and Peg13 IncRNAs that were identified by RNA-Seq and Fam2019aos, Platr28, and Neat1 IncRNAs that were identified through microarray hybridizations (Supplemental material Figure 2).

\section{Discussion}

The autoimmune regulator (AIRE) protein complex, which is formed in the nucleus by the interactions between AIRE and its partner proteins (Abramson et al., 2010; Kyewski and Peterson, 2010), exerts its function by advancing stalled RNA Pol II on chromatin, thereby enabling the transcription elongation phase in which both coding and noncoding RNAs are synthesized (Giraud et al., 2012; Waterfield et al., 2014; Passos et al., 2018). This property guided us when we observed that the Aire gene could control miRNAs in medullary thymic epithelial cells (mTECs) (Macedo et al., 2013).

A possible association of Aire with posttranscriptional control in mTECs began to be considered in our group when we discussed the results obtained by the Giraud's group (Giraud et al., 2012). There are findings indicating that AIRE protein pushes stalled RNA Pol II on chromatin. Since RNA Pol II transcribes miRNAs in addition to mRNAs, Aire might even indirectly control the expression of miRNAs (Macedo et al., 2013).

Given this possibility, we hypothesized that Aire could control IncRNAs in mTECs. As the link between mTECs, Aire and IncRNAs has not been explored to date, we realize the uniqueness of this proposition. Therefore, we established a model system that makes it possible to test this hypothesis. The primary mTEC 3.10 cell line of continuous growth in culture (Hirokawa et al., 1986; Mizuochi et al., 1992) and its Aire KO subclone (Aire ${ }^{-/}$mTEC 3.10E6) (Speck-Hernandez et al., 2018).

The in vitro adhesion with thymocytes significantly modulates the transcriptional activity of mTECs, upregulating the transcriptional expression of Aire and Fezf2, as well as cell adhesion-related genes such as Cd80 or Tcf7, among others (Speck-Hernandez et al., 2018; Cotrim-Sousa et al., 2019). Therefore, we ponder that the mTEC-thymocyte coculture comprises a model system, which is helpful for large-scale transcriptional gene expression studies influenced or not influenced by adhesion with thymocytes.

Considering that adhesion with thymocytes is an essential property of mTECs, we evaluated this variable because it also controls transcriptional gene expression. Compared to Aire wild-type mTECs, the Aire KO mTECs adhere less to the thymocytes of $\mathrm{CD}^{+}$or $\mathrm{CD}^{+}$phenotypes, which corroborated our previous findings (SpeckHernandez et al., 2018) and validated the model system for this study.

The prepared RNA-Seq library enabled us to evaluate the IncRNA sequences that are differentially expressed when comparing Aire wild-type vs. Aire KO mTECs 
before or after thymocyte adhesion. This finding represents the first observation that Aire can control IncRNAs in mTECs with synergistic adhesion action with thymocytes. Therefore, the IncRNAs influenced by Aire are referred to in this report as Airedependent. Among the set of differentially expressed (DE) IncRNAs, also were found the sub-class of intergenic IncRNAs (lincRNAs) (Ransohoff et al., 2018). The IncRNA Ifi30 is Aire-dependent and is of particular interest, since it participates in antigen processing and presentation pathways (https://www.genome.jp/kegg/pathway.html); it might contribute to mTECs during self-antigen presentation.

It was possible to determine the chromosomal location of the differentially expressed IncRNAs and their closest coding genes. The genomic location is essential to predict the mode of action of IncRNAs; in other words, the IncRNAs can either act in cis, regulating genes close to their locus on the same chromosome, or in trans, when they regulate genes located in distant regions on different chromosomes (Gil and Ulitsky, 2020; Yan et al., 2017). We found that the IncRNAs Fendrr, Gas5, Malat1, Neat1, and Xist might, in the model system studied, act in cis, since their closest protein-encoding targets are located on the same DNA segment on chromosome either upstream or downstream (Bao et al., 2019).

The expression levels of the IncRNAs Fendrr, and Peg13, as measured by RNASeq, were further validated using RT-qPCR. The results obtained in this experiment corroborated the bioinformatic analysis of the RNA-seq dataset, confirming the quality and precision of the in silico analyses. Fendrr is one of the most well-characterized IncRNA at present (Grote, 2014). It has been shown that Fendrr acts in the development of the heart of mice and that its downregulation leads to heart failure (Grote, 2014). In human gastric cancer and renal carcinoma, Fendrr upregulation has been reported to be associated with a worse prognosis, as it promotes increased migration, proliferation, and invasion of cancer cells (He et al., 2019).

Therefore, this study is the first that associates the IncRNA Fendrr with the immune system, i.e., thymic stromal cells. It is worth pursuing this IncRNA to determine if, in the mTEC cells, it is involved in proliferation and/or migration processes that are essential in the biology of the thymus.

In previous studies performed in our laboratory, it was observed that both silencing or knocking out the Aire gene in MTEC 3.10 cells resulted in a decrease in the ability of these cells to adhere to thymocytes (Pezzi et al., 2016; Speck-Hernandez et al., 2018). Genes involved in cell-cell adhesion, such as Cd80, Itgam, Tgfbi, Cdh16 and Itgae had their expression levels reduced when Aire was disrupted.

Considering that the physical process of interaction between thymocytes and mTECs is crucial in presenting PTAs and the maturation of mTECs and T-cells themselves, the absence of Aire alters the entire connection structure, compromising the functioning of central immune tolerance (Derbinski et al., 2005; Passos et al., 2018; Perniola, 2018).

One crucial point is that mTEC-thymocyte crosstalk can influence the transcriptional profile of mRNAs as previously reported (Speck-Hernandez et al., 2018; Cotrim-Sousa et al., 2019) and in this study we hypothesized that IncRNAs might be 
modulated in such condition. Through the adhesion assay, it was possible to observe that in the culture of Aire KO mTEC 3.10E6 cells with thymocytes, varying the coculture time between 12 or $36 \mathrm{~h}$, there was a decrease in adhesion compared with WT MTEC 3.10 cells. We found no cell morphology changes in visual observation and under an optical microscope between WT mTEC 3.10 cells and Aire KO mTEC $3.10 E 6$ cells.

We were able in demonstrating that thymocyte adhesion also modulates IncRNAs in mTECs. After coculturing Aire WT mTEC 3.10 cells with thymocytes, we identified 191 differentially expressed IncRNAs, 53 after $12 \mathrm{~h}$ and 138 after $36 \mathrm{~h}$ of coculture. Among these RNAs, we focused on the IncRNA NEAT1, which plays a role in controlling the formation of paraspeckle heterochromatin structures (Clemson et al., 2009; Zhang et al., 2013). This result suggests that longer thymocyte adhesion stimulates IncRNA-mediated posttranscriptional control in mTECs.

During T-cell development, immature T-cells interact with the cell matrix of the thymus, which is composed primarily of type I and IV collagen, integrins, laminin, and fibronectin (Savino et al., 2004). Integrins are involved in the interaction with the extracellular matrix, physical cell-cell contact, and adhesion receptors. Integrins are essential in the processes that modulate the proliferation and differentiation of T-cells (Savino et al., 2004, 2013; Bertoni et al., 2018).

Interestingly, the deregulation of transcriptional expression of IncRNAs was observed in Aire KO mTECs, suggesting the involvement of Aire-IncRNAs axis with the expression of molecules linked to cell-cell and cell-matrix interactions.

Although not explored in this study, there may be posttranscriptional interaction between mRNAs that encode cell adhesion proteins and IncRNAs identified here.

To compare the extent to which IncRNAs are controlled by Aire in an in vivo system, we reanalyzed raw RNA-seq data previously collected from mTECs (mTEC ${ }^{\text {high }}$ and TTEC $^{\text {low }}$ ) from Aire WT and Aire KO mice (St Pierre et al., 2015 ). It was possible to observe that both in an in vitro and in vivo model systems, there was modulation of IncRNAs using the approach of loss-of-function (LOF) of Aire (Aire KO). The mTEC subtypes that most expressed exclusive IncRNAs were Aire KO mTEC ${ }^{\text {high }}$ (in vivo) and Aire KO MTEC 3.10E6 (in vitro without adhesion with thymocytes). In addition, subsets of exclusive and shared IncRNAs were also observed between Aire KO mTEC low (in vivo), Aire KO mTEC high (in vivo), Aire KO MTEC 3.10E6 (in vitro without adhesion) and Aire KO mTEC 3.10E6 (in vitro under adhesion with thymocytes).

In conclusion, the results of this study demonstrate for the first time the existence of a link between Aire and the expression IncRNAs in mTEC cells. The adhesion between mTECs and thymocytes was shown to be synergistic in this process. Through the knockout of Aire, we were able to employ a LOF strategy and observe that downstream IncRNAs are deregulated. 
Considering the regulatory role of IncRNAs, the axis Aire-IncRNAs in mTECs found in this study, might be important for the in vivo induction of central immunological tolerance.

\section{Competing Interests}

The authors declare that there are no competing interests associated with the manuscript.

\section{Data availability}

High-quality paired-end reads of this study are available at the Gene Expression Omnibus (GEO) database under accession number GSE91015. The microarray data are available at the ArrayExpress database under accession number E-MTAB-6541.

\section{Acknowledgements}

We thank Dr. Wilson Savino (Laboratory on Thymus Research, Oswaldo Cruz Institute, Fiocruz, Rio de Janeiro, Brazil) that gently ceded the Aire WT mTEC 3.10 line, Dr. Daniella A. Mendes-da-Cruz and Dr. Vinícius Cotta-Almeida (Laboratory on Thymus Research, Oswaldo Cruz Institute, Fiocruz, Rio de Janeiro, Brazil) and Dr. Eduardo A. Donadi (Ribeirão Preto Medical School, USP, Ribeirão Preto, Brazil) for help and discussions and MSc Denise B. Ferraz (Ribeirão Preto Medical School, USP, Ribeirão Preto, Brazil) for technical assistence.

This study was funded by São Paulo Research Foundation (Fapesp, São Paulo, Brazil, through grants \# 13/17481-1 and \# 17/10780-4 to GAP), Conselho Nacional de Desenvolvimento Científico e Tecnológico (CNPq, Brasília, Brazil, through grant \# 305787/2017-9 to GAP). This study was financed in part by the Coordenação de Aperfeiçoamento de Pessoal de Nível Superior (CAPES, Brasília, Brazil) through Financial Code 001.

\section{References}

Abramson, J., et al., (2010). Aire's partners in the molecular control of immunological tolerance. Cell, 140, 123-135. https://doi.org/10.1016/j.cell.2009.12.030

Assis, A.F., et al., (2014). What is the transcriptome and how it is evaluated? In: Transcriptomics in Health and Disease (1st ed.). Springer. doi: 10.1007/978-3-31911985-4_1

Bao, Z., et al., (2019). LncRNADisease 2.0: an updated database of long non-coding RNA-associated diseases. Nucleic acids research, 47, D1034-D1037. https://doi.org/10.1093/nar/gky905

Bertoni, A., et al., (2018). Integrins in T Cell Physiology. International journal of molecular sciences, 19, 485. https://doi.org/10.3390/ijms19020485 
Bhan, A., et al., (2017). Long Noncoding RNA and Cancer: A New Paradigm. Cancer research, 77, 3965-3981. https://doi.org/10.1158/0008-5472.CAN-16-2634

Bhatti, G. K., et al., (2021a) Emerging role of non-coding RNA in health and disease. Metab Brain Dis (online ahead of print) https://doi.org/10.1007/s11011-021-00739-y

Bhatti, Z., et al., (2021b) The nuclear functions of long noncoding RNAs come into focus. Noncoding RNA Res 6:70-79 . https://doi.org/10.1016/j.ncrna.2021.03.002

Chen, Q. N., et al., (2017). Long non-coding RNAs in anti-cancer drug resistance. Oncotarget, 8, 1925-1936. https://doi.org/10.18632/oncotarget.12461

Clemson, C. M., et al., (2009). An architectural role for a nuclear noncoding RNA: NEAT1 RNA is essential for the structure of paraspeckles. Molecular cell, 33, 717-726. https://doi.org/10.1016/j.molcel.2009.01.026

Cotrim-Sousa, L., et al., (2019). Adhesion between medullary thymic epithelial cells and thymocytes is regulated by miR-181b-5p and miR-30b. Molecular immunology, 114, 600-611. https://doi.org/10.1016/j.molimm.2019.09.010

Derbinski, J., et al., (2005). Promiscuous gene expression in thymic epithelial cells is regulated at multiple levels. The Journal of experimental medicine, 202, 33-45. https://doi.org/10.1084/jem.20050471

Donate, P. B., et al., (2013). T cell post-transcriptional miRNA-mRNA interaction networks identify targets associated with susceptibility/resistance to collagen-induced arthritis. PloS one, 8, e54803. https://doi.org/10.1371/journal.pone.0054803

Fornari, T. A., et al., (2015). Comprehensive Survey of miRNA-mRNA Interactions Reveals That Ccr7 and Cd247 (CD3 zeta) are Posttranscriptionally Controlled in Pancreas Infiltrating T Lymphocytes of Non-Obese Diabetic (NOD) Mice. PloS one, 10, e0142688. https://doi.org/10.1371/journal.pone.0142688

Gil, N., Ulitsky, I. (2020). Regulation of gene expression by cis-acting long non-coding RNAs. Nature reviews. Genetics, 21, 102-117. https://doi.org/10.1038/s41576-0190184-5

Giraud, M., et al., (2012). Aire unleashes stalled RNA polymerase to induce ectopic gene expression in thymic epithelial cells. Proceedings of the National Academy of Sciences of the United States of America, 109, 535-540. https://doi.org/10.1073/pnas.1119351109

Grote, P., et al., (2013). The tissue-specific IncRNA Fendrr is an essential regulator of heart and body wall development in the mouse. Developmental cell, 24, 206-214. https://doi.org/10.1016/j.devcel.2012.12.012

He, W., et al., (2019). Downregulation of long noncoding RNA FENDRR predicts poor prognosis in renal cell carcinoma. Oncology letters, 17, 103-112. https://doi.org/10.3892/ol.2018.9624 
Hirokawa, K., et al., (1986). Analysis of the thymic microenvironment by monoclonal antibodies with special reference to thymic nurse cells. Thymus, 8, 349-360.

Huang, D., et al., (2009). Bioinformatics enrichment tools: paths toward the comprehensive functional analysis of large gene lists. Nucleic acids research, 37, 1-13. https://doi.org/10.1093/nar/gkn923

Jarroux, J., et al., (2017). History, Discovery, and Classification of IncRNAs. Advances in experimental medicine and biology, 1008, 1-46. https://doi.org/10.1007/978-981-105203-3_1

Kang, Y. J., et al., (2017). CPC2: a fast and accurate coding potential calculator based on sequence intrinsic features. Nucleic acids research, 45, W12-W16. https://doi.org/10.1093/nar/gkx428

Kauffmann, A., et al., (2009). arrayQualityMetrics--a bioconductor package for quality assessment of microarray data. Bioinformatics (Oxford, England), 25, 415-416. https://doi.org/10.1093/bioinformatics/btn647

Kong, L., et al., (2007). CPC: assess the protein-coding potential of transcripts using sequence features and support vector machine. Nucleic acids research, 35, W345W349. https://doi.org/10.1093/nar/gkm391

Kyewski, B., Peterson, P. (2010). Aire, master of many trades. Cell, 140, 24-26. https://doi.org/10.1016/j.cell.2009.12.036

Li, B., et al., (2021) Comprehensive analysis of IncRNAs, miRNAs and mRNAs related to thymic development and involution in goose. Genomics 113: 1176-1188 https://doi.org/10.1016/j.ygeno.2020.11.031

López-Romero, P., et al., (2010). Processing of Agilent microRNA array data. BMC research notes, 3, 18. https://doi.org/10.1186/1756-0500-3-18

Macedo, C., et al., (2013). Autoimmune regulator (Aire) controls the expression of microRNAs in medullary thymic epithelial cells. Immunobiology, 218, 554-560. https://doi.org/10.1016/j.imbio.2012.06.013

Mathy, N. W., Chen, X. M. (2017). Long non-coding RNAs (IncRNAs) and their transcriptional control of inflammatory responses. The Journal of biological chemistry, 292, 12375-12382. https://doi.org/10.1074/jbc.R116.760884

Messias, C. V., et al., (2020) Zika virus targets the human thymic epithelium. Sci. Rep. https://doi.org/10.1038/s41598-020-58135-y

Mizuochi, T., et al., (1992). Medullary but not cortical thymic epithelial cells present soluble antigens to helper T cells. The Journal of experimental medicine, 175, 16011605. https://doi.org/10.1084/jem.175.6.1601 
Nihei, O. K., et al., (2003). A novel form of cellular communication among thymic epithelial cells: intercellular calcium wave propagation. American journal of physiology. Cell physiology, 285, C1304-C1313. https://doi.org/10.1152/ajpcell.00568.2002

Oliveira, E. H., et al., (2016). Aire Downregulation Is Associated with Changes in the Posttranscriptional Control of Peripheral Tissue Antigens in Medullary Thymic $\begin{array}{llll}\text { Epithelial Cells. Frontiers in } & \end{array} 26$. https://doi.org/10.3389/fimmu.2016.00526

Oliveira, E. H., et al., (2013). Expression profile of peripheral tissue antigen genes in medullary thymic epithelial cells (mTECs) is dependent on mRNA levels of autoimmune regulator (Aire). Immunobiology, 218, 96-104. https://doi.org/10.1016/j.imbio.2012.02.005

Passos, G. A., et al., (2018). Update on Aire and thymic negative selection. Immunology, 153, 10-20. https://doi.org/10.1111/imm.12831

Passos, G. A., et al., (2015). The Thymic Orchestration Involving Aire, miRNAs, and CellCell Interactions during the Induction of Central Tolerance. Frontiers in immunology, 6, 352. https://doi.org/10.3389/fimmu.2015.00352

Perniola R. (2018). Twenty Years of AIRE. Frontiers in immunology, 9, 98. https://doi.org/10.3389/fimmu.2018.00098

Pezzi, N., et al., (2016). Aire knockdown in medullary thymic epithelial cells affects Aire protein, deregulates cell adhesion genes and decreases thymocyte interaction. Molecular immunology, 77, 157-173. https://doi.org/10.1016/j.molimm.2016.08.003

Ribeiro, C., et al., (2019) Medullary thymic epithelial cells: Deciphering the functional diversity beyond promiscuous gene expression. Immunol lett, 215, 24-27. doi: 10.1016/j.imlet.2019.01.012

Ransohoff, J. D., et al., (2018). The functions and unique features of long intergenic non-coding RNA. Nature reviews. Molecular cell biology, 19, 143-157. https://doi.org/10.1038/nrm.2017.104

Savino W. (2010). Intrathymic T cell migration is a multivectorial process under a complex neuroendocrine control. Neuroimmunomodulation, 17, 142-145. https://doi.org/10.1159/000258708

Savino, W., et al., (2004). Molecular mechanisms governing thymocyte migration: combined role of chemokines and extracellular matrix. Journal of leukocyte biology, 75, 951-961. https://doi.org/10.1189/jlb.1003455

Savino, W., et al., (2003). Thymocyte migration: an affair of multiple cellular interactions? Brazilian journal of medical and biological research 36, 1015-1025. https://doi.org/10.1590/s0100-879x2003000800007 
Speck-Hernandez, C. A., et al., (2018). Aire Disruption Influences the Medullary Thymic Epithelial Cell Transcriptome and Interaction With Thymocytes. Frontiers in immunology, 9, 964. https://doi.org/10.3389/fimmu.2018.00964

Su, Y., et al., (2020) IncRNAs classifier to accurately predict the recurrence of thymic epithelial tumors. Thorac Cancer 11:1773-1783 https://doi.org/10.1111/17597714.13439

St-Pierre C., et al (2015) Differential Features of AIRE-Induced and AIRE-Independent Promiscuous Gene Expression in Thymic Epithelial Cells. J Immunol, 195, 498-506. doi: 10.4049/jimmunol.1500558

Takaba, H., Takayanagi, H. (2017). The Mechanisms of T Cell Selection in the Thymus. Trends in immunology, 38, 805-816. https://doi.org/10.1016/j.it.2017.07.010

Takaba, H., et al., (2015). Fezf2 Orchestrates a Thymic Program of Self-Antigen Expression for Immune Tolerance. Cell, 163, 975-987. https://doi.org/10.1016/j.cell.2015.10.013

Tomofuji, Y., et al., (2020). Chd4 choreographs self-antigen expression for central immune tolerance. Nature Immunology, 21, 892-901. https://doi.org/10.1038/s41590020-0717-2

Ucar, O., et al., (2013). An evolutionarily conserved mutual interdependence between Aire and microRNAs in promiscuous gene expression. European Journal of Immunology, 43, 1769-1778. https://doi.org/10.1002/eji.201343343

Waterfield, M., et al., (2014). The transcriptional regulator Aire coopts the repressive ATF7ip-MBD1 complex for the induction of immunotolerance. Nature immunology, 15, 258-265. https://doi.org/10.1038/ni.2820

Wei, C., et al., (2018). Profiling analysis of $17 \beta$-estradiol-regulated IncRNAs in mouse thymic epithelial cells. Physiological genomics, 50, 553-562. https://doi.org/10.1152/physiolgenomics.00098.2017

Wettenhall, J. M., Smyth, G. K. (2004). limmaGUI: a graphical user interface for linear modeling of microarray data. Bioinformatics (Oxford, England), 20, 3705-3706. https://doi.org/10.1093/bioinformatics/bth449

Yan, P., et al., (2017). Cis- and trans-acting IncRNAs in pluripotency and reprogramming. Current opinion in genetics \& development, 46, 170-178. https://doi.org/10.1016/j.gde.2017.07.009

Yao, R. W., et al., (2019). Cellular functions of long noncoding RNAs. Nature cell biology, 21, 542-551. https://doi.org/10.1038/s41556-019-0311-8

Zemmour, D., et al., (2017). Flicr, a long noncoding RNA, modulates Foxp3 expression and autoimmunity. Proceedings of the National Academy of Sciences of the United States of America, 114, E3472-E3480. https://doi.org/10.1073/pnas.1700946114 
bioRxiv preprint doi: https://doi.org/10.1101/2021.07.14.452339; this version posted July 14, 2021. The copyright holder for this preprint (which was not certified by peer review) is the author/funder, who has granted bioRxiv a license to display the preprint in perpetuity. It is made available under aCC-BY-NC-ND 4.0 International license.

Zhang, Q., et al., (2013). NEAT1 long noncoding RNA and paraspeckle bodies modulate HIV-1 posttranscriptional expression. mBio, 4, e00596-12. https://doi.org/10.1128/mBio.00596-12

Zhang, Y., et al., (2018). Long noncoding RNA: a crosslink in biological regulatory network. Briefings in bioinformatics, 19, 930-945. https://doi.org/10.1093/bib/bbx042

\section{SUPPLEMENTAL MATERIAL FIGURE 1}

A

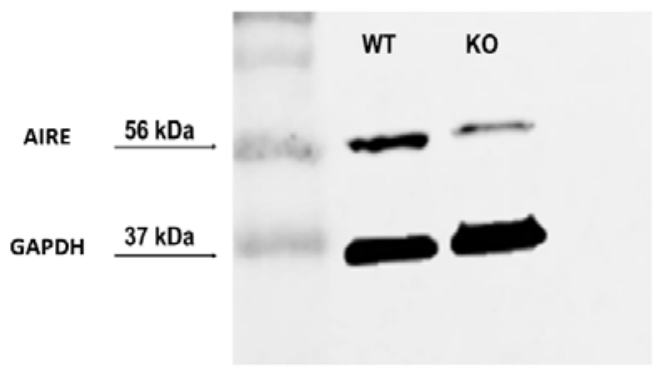

B

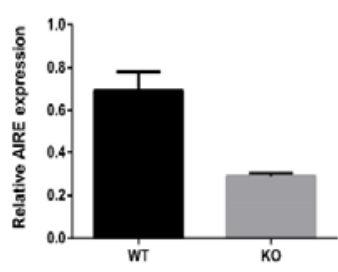

Supplemental material Figure 1 (A) Representative western blot after SDS-PAGE of Aire WT mTEC 3.10 and Aire KO mTEC 3.10E6 cell lysates, probed with an antibody against AIRE protein. GAPDH protein was used as an internal load control. (B) Western blot bands were quantified by ImageJ software, and AIRE protein levels (normalized to GAPDH) were expressed as signal intensity (pixels/area). The bar graph shows that the defective mutant AIRE protein is expressed at low levels in the Aire KO mTEC 3.10E6 cell line. The cell lysates were prepared from Aire WT mTEC 3.10 or Aire KO mTEC $3.10 \mathrm{E} 6$ cell lines with no adhesion with thymocytes. 


\section{SUPPLEMENTAL MATERIAL FIGURE 2}

A

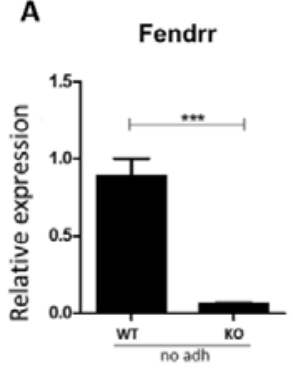

C

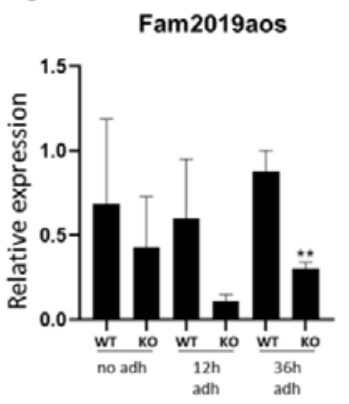

B

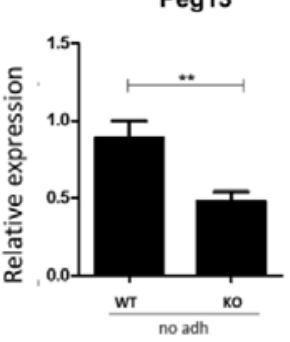

D

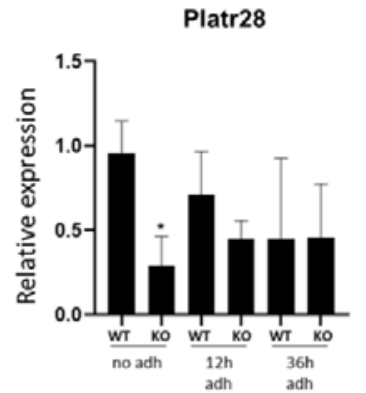

$\mathbf{E}$

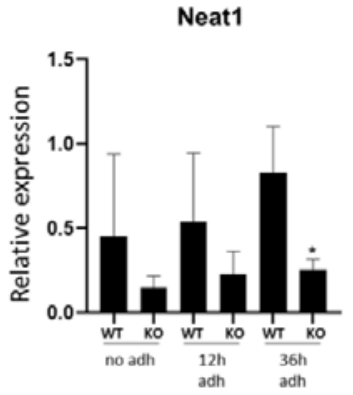

Supplemental material Figure 2 Validation of the relative transcriptional expression levels of the Fendrr, Peg13, Fam219aos, Platr28, Neat1 IncRNAs (A-E), comparing Aire wild-type (WT) mTEC 3.10 with Aire KO MTEC 3.10E6 before and after thymocyte adhesion. The transcriptional expression of IncRNAs were quantified by real-time quantitative-PCR (RT-qPCR). Means \pm S.D. from experiments from three independent replicates. $P<0.05$. 


\begin{tabular}{llll}
\hline ID_EnsembI & IncRNA & Log2 (Fold Change) & FDR \\
\hline ENSMUSG00000104811.1 & Gm33093 & 3.05697094892126 & $1.68887769298415 \mathrm{e}-11$ \\
ENSMUSG00000097289.1 & 2010300F17Rik & 2.80000087711663 & $1.49799978763693 \mathrm{e}-69$ \\
ENSMUSG00000113185.1 & Gm47127 & 2.76977635492375 & $4.10197679502256 \mathrm{e}-07$ \\
ENSMUSG00000109021.1 & Gm44812 & 1.98691880403124 & 0.000662818348209971 \\
ENSMUSG00000104554.1 & Gm4610 & 1.86186727758227 & $1.39654733697852 \mathrm{e}-22$ \\
ENSMUSG00000097636.7 & Mirt1 & 1.82963943266738 & $6.24891059179398 \mathrm{e}-73$ \\
ENSMUSG00000104982.1 & Gm32554 & 1.7898999306723 & $2.03458606660714 \mathrm{e}-15$ \\
ENSMUSG00000106121.1 & Gm42679 & 1.72570384462814 & 0.000251621874711925 \\
ENSMUSG00000087107.8 & Al662270 & 1.687331514335 & $5.97330879447768 \mathrm{e}-06$
\end{tabular}

Supplementary Table 1 Top 10 upregulated differentially expressed IncRNAs in Aire KO mTECs.

SUPPLEMENTAL MATERIAL TABLE 1, TABLE 2, TABLE 3 


ENSMUSG00000106652.1 Gm43358 $\quad 1.52897408641536 \quad 0.000789113531882971$

\begin{tabular}{llll}
\hline $\begin{array}{l}\text { Supplementary Table 1.1 Top 10 downregulated differentially expressed IncRNAs in Aire KO } \\
\text { mTECs. }\end{array}$ & & \multicolumn{1}{c}{ FDR } \\
\hline ID_Ensembl & IncRNA & Log2 (Fold Change) & \multicolumn{1}{c}{. } \\
\hline ENSMUSG00000097336.8 & Fendrr & -4.39841211281411 & $2.9813879363468 \mathrm{e}-20$ \\
ENSMUSG00000086324.8 & Gm15564 & -2.8992396959009 & 0.000588293004549254 \\
ENSMUSG00000110537.1 & Gm4316 & -1.92139045902179 & 0.00778888982304384 \\
ENSMUSG00000051832.7 & E230016K23Rik & -1.84972000470977 & $7.52183360620377 \mathrm{e}-05$ \\
ENSMUSG00000087132.7 & A930001C03Rik & -1.6690622794737 & 0.000788398326160964 \\
ENSMUSG00000112627.1 & 4933412 E12Rik & -1.54359798447323 & $2.4157528207743 \mathrm{e}-05$ \\
ENSMUSG00000048603.8 & Gm9828 & -1.52886402885372 & 0.00707536255075073 \\
ENSMUSG00000086629.8 & $2810403 \mathrm{D} 21$ Rik & -1.51183828152075 & 0.000119022734938128 \\
ENSMUSG00000057359.7 & Gm17494 & -1.50537074849227 & 0.00250079518114827 \\
ENSMUSG00000085147.1 & Gm12609 & -1.4788977971886 & 0.0037369013076587
\end{tabular}


Supplementary Table 2 Nearby protein coding genes to the differentially expressed IncRNAs.

\begin{tabular}{|c|c|c|c|c|}
\hline \multicolumn{5}{|c|}{ Nearby protein coding genes to IncRNAs (no adhesion to thymocytes) } \\
\hline Distance (pb) & Downstream & IncRNA & Upstream & Distance (bp) \\
\hline 395798 & Zfp516 & 4921531P14Rik & Tshz1 & 779943 \\
\hline 5558 & Josd2 & 5430431A17Rik & Emc10 & 22991 \\
\hline 98339 & Mogat1 & 5730419F03Rik & Acsl3 & 48317 \\
\hline 627451 & Rad51b & 9430078K24Rik & Zfp36I1 & 188280 \\
\hline 346674 & Irf8 & Fendrr & Foxf1 & 1354 \\
\hline 36541 & Dnajb11 & Gm30505 & Ahsg & -3109 \\
\hline 95399 & Ube3a & Snhg14 & Gm38393 & 680915 \\
\hline 61244 & Gm26992 & Xist & Zcchc13 & 147332 \\
\hline 687265 & Lhfpl3 & 5031425E22Rik-202 & $\mathrm{Kmt2e}$ & 1117 \\
\hline 539380 & Etaa1 & Gm12022-201 & Meis1 & 525730 \\
\hline 238606 & A1bg & Gm38563-201 & Myc & 827522 \\
\hline 33577 & $\mathrm{Rbm} \times 12$ & Platr28-201 & Syt9 & 127706 \\
\hline 23651 & 4930572003Rik & Speer4cos-201 & Speer4c & 33526 \\
\hline
\end{tabular}

Supplementary Table 2.1 Nearby protein coding genes to the differentially expressed IncRNAs.

\begin{tabular}{lllll}
\hline \multicolumn{4}{c}{ NEARBY PROTEIN CODING GENES TO LNCRNAS (12 H ADHESION TO THYMOCYTES) } \\
\hline Distance (pb) & Downstream & Incrna & Upstream & Distance (bp) \\
\hline 631 & Serpinb6a & 1110046J04Rik & Nqo2 & 29120 \\
28391 & Trpv2 & 2410006H16Rik & Lrrc75a & 45646 \\
61938 & Dmrt3 & 2610016A17Rik & Dmrt2 & 172 \\
113190 & P4ha2 & 4933405E24Rik & Csf2 & 36382 \\
46586 & Rex2 & 4933438K21Rik & Zfp991 & 63602 \\
5558 & Josd2 & 5430431A17Rik & Emc10 & 22991 \\
98339 & Mogat1 & 5730419F03Rik & Acsl3 & 48317 \\
627451 & Rad51b & 9430078K24Rik & Zfp36l1 & 188280 \\
121392 & Avpr1a & A130077B15Rik & Ppm1h & 108871 \\
173022 & S1pr1 & A930005H10Rik & Dph5 & -257 \\
32246 & Slfn3 & Al662270 & Slfn14 & 63150 \\
96509 & Atp6v0a2 & Dnah10 & Ccdc92 & 137339 \\
26167 & Zfp846 & Fbxl12os & Fbxl12 & 34855 \\
346674 & Irf8 & Fendrr & Foxf1 & 1354 \\
539380 & Etaa1 & Gm12022 & Meis1 & 525730 \\
24137 & Stn1 & Gm19557 & Slk & 66696 \\
238606 & A1bg & Gm38563 & Myc & 827522 \\
22958 & Dnajc19 & Sox2ot & Sox2 & 546135 \\
23651 & 4930572O03Rik & Speer4cos & Speer4c & 33526 \\
21058 & Crybg1 & Speer5-ps1 & Atg5 & 98447 \\
116851052 & Gm27236 & Speer6-ps1 & Gdi2 & 388588 \\
13246 & Gm4792 & Tmcc3 & Cep83 & 376705 \\
61244 & Gm26992 & Xist & Zcchc13 & 147332 \\
\hline & & & &
\end{tabular}


Supplementary Table 2.2 Nearby protein coding genes to the differentially expressed IncRNAs.

NEARBY PROTEIN CODING GENES TO LNCRNAS (36 H ADHESION TO THYMOCYTES)

\begin{tabular}{|c|c|c|c|c|}
\hline Distance (pb) & Downstream & Lncrna & Upstream & Distance (bp) \\
\hline 631 & Serpinb6a & 1110046J04Rik & $\mathrm{NqO} 2$ & 29120 \\
\hline 87324 & Gm8922 & 1700003C15Rik & Gm8926 & 1265 \\
\hline 2160883 & Pabpc4l & 1700018B24Rik & Pcdh18 & 1148214 \\
\hline 2428 & 1700018B08Rik & 1700030M09Rik & Fbxo31 & 34424 \\
\hline 34384 & Ubash3b & 1700063D05Rik & Sorl1 & 931851 \\
\hline 1574 & Esrp2 & 1810019D21Rik & Pla2g15 & 14999 \\
\hline 194 & Sap30 & 2500002B13Rik & Hmgb2 & 23853 \\
\hline 61938 & Dmrt3 & 2610016A17Rik & Dmrt2 & 172 \\
\hline 455521 & Snx29 & 4833415N18Rik & Cpped1 & 130994 \\
\hline 113190 & P4ha2 & 4933405E24Rik & Csf2 & 36382 \\
\hline 46586 & $\operatorname{Rex} 2$ & 4933438K21Rik & Zfp991 & 63602 \\
\hline 5558 & Josd2 & 5430431A17Rik & Emc10 & 22991 \\
\hline 98339 & Mogat1 & 5730419F03Rik & Acsl3 & 48317 \\
\hline 2741 & 4930412013Rik & 9230102004Rik & Taf3 & 69664 \\
\hline 627451 & Rad51b & 9430078K24Rik & Zfp36l1 & 188280 \\
\hline 136752 & Ercc3 & A830052D11Rik & Bin1 & 178 \\
\hline 4317 & $\mathrm{Nr} 2 \mathrm{f} 2$ & B130024G19Rik & Mctp2 & 1941698 \\
\hline 4043 & Smim40 & BC051226 & Daxx & 236 \\
\hline 96509 & Atp6v0a2 & Dnah10 & Ccdc92 & 137339 \\
\hline 3420 & Ccl9 & E230016K23Rik & Ccl6 & 11031 \\
\hline 9683 & 1110017D15Rik & Fam219aos & Fam219a & 52072 \\
\hline 26167 & Zfp846 & Fbxl12os & Fbxl12 & 34855 \\
\hline 80 & Fkbp4 & Gm10069 & Pzp & 87963 \\
\hline 539380 & Etaa1 & Gm12022 & Meis1 & 525730 \\
\hline 156583 & $\mathrm{HaO} 2$ & Gm12440 & Wars2 & 91246 \\
\hline 110809 & Cdkn2b & Gm12610 & Dmrta1 & 257588 \\
\hline 705861 & Dipk2b & Gm14636 & Tex13c3 & 314087 \\
\hline 34937 & Atg2a & Gm14963 & Ehd1 & 120 \\
\hline 78433 & Cacna1s & Gm15850 & Kif21b & 206 \\
\hline 64 & Flcn & Gm16062 & Cops3 & 8607 \\
\hline 42429 & Fgfr1 & Gm16159 & Letm2 & 41430 \\
\hline 13054 & Tcf20 & Gm20324 & Nfam1 & 134226 \\
\hline 181118 & Zcchc2 & Gm20753 & Phlpp1 & 228 \\
\hline 36541 & Dnajb11 & Gm30505 & Ahsg & -3109 \\
\hline 16850 & $\mathrm{Bcl} 7 \mathrm{c}$ & Gm39090 & Ctf1 & 20801 \\
\hline 27079 & Kcnk12 & Gm50039 & Rpl36-ps4 & 95904 \\
\hline 190187 & Kcnq1 & Kcnq1ot1 & Cdkn1c & 164447 \\
\hline 88178 & Dlk1 & Meg3 & Rt|1 & 59329 \\
\hline 169015 & Ubash3b & Mir100hg & Sorl1 & 797220 \\
\hline 7101 & Wdr81 & Mir22hg & Tlcd2 & 176 \\
\hline 74059 & Scyl1 & Neat1 & Frmd8 & 29796 \\
\hline 39306 & Cstb & $\mathrm{Pdxk}$ & Olfr1358 & 52453 \\
\hline
\end{tabular}


bioRxiv preprint doi: https://doi.org/10.1101/2021.07.14.452339; this version posted July 14, 2021. The copyright holder for this preprint (which was not certified by peer review) is the author/funder, who has granted bioRxiv a license to display the preprint in perpetuity. It is made available under aCC-BY-NC-ND 4.0 International license.

\begin{tabular}{lllll}
83471 & Gne & Rnf38 & Melk & 133228 \\
153795 & Ryk & Slco2a1 & Rab6b & 123075 \\
210304 & Sil1 & Snhg4 & Matr3 & 8767 \\
22958 & Dnajc19 & Sox2ot & Sox2 & 546135 \\
23651 & 4930572003Rik & Speer4cos & Speer4c & 33526 \\
21058 & Crybg1 & Speer5-ps1 & Atg5 & 98447 \\
116851052 & Gm27236 & Speer6-ps1 & Gdi2 & 388588 \\
13246 & Gm4792 & Tmcc3 & Cep83 & 376705 \\
61244 & Gm26992 & Xist & Zcchc13 & 147332 \\
22327 & Slfn8 & Slfn10-ps & Slfn2 & 25070 \\
\hline
\end{tabular}




\begin{tabular}{lll}
\hline InCRNA & Coding probability & Classification \\
\hline SNHG14 & 0.439997 & noncoding \\
FENDRR & 0.0656715 & noncoding \\
PLATR28 & 0.0305438 & noncoding \\
SPEER4COS-201 & 0.201759 & noncoding \\
4921531P14RIK & 0.0398758 & noncoding \\
5031425E22RIK & 0.424424 & noncoding \\
5430431A17RIK & 0.212594 & noncoding \\
5730419F03RIK & 0.030601 & noncoding \\
9430078K24RIK & 0.157714 & noncoding \\
GM12022-201 & 0.0201895 & noncoding \\
GM30505 & 0.103945 & noncoding \\
GM38563-201 & 0.0475214 & noncoding \\
XIST & 0.0241659 & noncoding \\
\hline
\end{tabular}

Supplementary Table 3 Coding potential of IncRNAs.

Supplementary Table 3.1 Coding potential of IncRNAs after $12 \mathrm{~h}$ adhesion to thymocytes.

\begin{tabular}{|c|c|c|}
\hline IncRNA & Coding probability & Classification \\
\hline 1110046J04RIK & 0.0752217 & noncoding \\
\hline 2410006H16RIK & 0.035433 & noncoding \\
\hline 2610016A17RIK & 0.0149574 & noncoding \\
\hline 4933405E24RIK & 0.0232256 & noncoding \\
\hline 4933438K21RIK & 0.0306206 & noncoding \\
\hline 5430431A17RIK & 0.212594 & noncoding \\
\hline 5730419F03RIK & 0.030601 & noncoding \\
\hline 9430078K24RIK & 0.157714 & noncoding \\
\hline A130077B15RIK & 0.11466 & noncoding \\
\hline A930005H10RIK & 0.231266 & noncoding \\
\hline Al662270 & 0.12172 & noncoding \\
\hline DNAH10-202 & 0.791989 & coding \\
\hline FBXL12OS & 0.185776 & noncoding \\
\hline FBXL12OS(REVERSE) & 0.257837 & noncoding \\
\hline FENDRR & 0.0656715 & noncoding \\
\hline GM12022-201 & 0.0201895 & noncoding \\
\hline GM19557 & 0.355901 & noncoding \\
\hline GM19557(REVERSE) & 0.32253 & noncoding \\
\hline GM38563-201 & 0.355901 & noncoding \\
\hline GM4262 & 0.0313553 & noncoding \\
\hline SOX2OT & 0.147919 & noncoding \\
\hline SPEER4COS-201 & 0.201759 & noncoding \\
\hline SPEER5-PS1-202 & 0.370882 & noncoding \\
\hline SPEER5-PS1-202(REVERSE) & 0.0102117 & noncoding \\
\hline
\end{tabular}


bioRxiv preprint doi: https://doi.org/10.1101/2021.07.14.452339; this version posted July 14, 2021. The copyright holder for this preprint (which was not certified by peer review) is the author/funder, who has granted bioRxiv a license to display the preprint in perpetuity. It is made available under aCC-BY-NC-ND 4.0 International license.

\begin{tabular}{llr} 
SPEER6-PS1-203 & 0.370882 & noncoding \\
TMCC3-206 & 0.0109869 & noncoding \\
XIST & 0.0241659 & noncoding \\
\hline
\end{tabular}

Supplementary Table 3.2 Coding potential of IncRNAs after $36 \mathrm{~h}$ adhesion to thymocytes.

\begin{tabular}{|c|c|c|}
\hline IncRNA & Coding probability & Classification \\
\hline 1110046J04Rik & 0.0752217 & noncoding \\
\hline 1700003C15Rik & 0.0418878 & noncoding \\
\hline 1700018B24Rik & 0.595009 & coding \\
\hline 1700030M09Rik & 0.0290032 & noncoding \\
\hline 1700063D05Rik & 0.0000117516 & noncoding \\
\hline 1810019D21Rik & 0.0222635 & noncoding \\
\hline 2500002B13Rik & 0.101008 & noncoding \\
\hline 2610016A17Rik & 0.0149574 & noncoding \\
\hline 4833415N18Rik & 0.130809 & noncoding \\
\hline 4930542C21Rik & 0.0281985 & noncoding \\
\hline 4933405E24Rik & 0.0232256 & noncoding \\
\hline 4933438K21Rik & 0.0701577 & noncoding \\
\hline 5430431A17Rik & 0.212594 & noncoding \\
\hline 5730419F03Rik & 0.030601 & noncoding \\
\hline A830052D11Rik & 0.0141421 & noncoding \\
\hline B130024G19Rik & 0.266976 & noncoding \\
\hline BC051226 & 0.0939098 & noncoding \\
\hline Dnah10-202 & 0.791989 & coding \\
\hline E230016K23Rik & 0.64385 & coding \\
\hline Fam219aos & 0.484752 & noncoding \\
\hline Fbxl12os & 0.185776 & noncoding \\
\hline Gm10069 & 0.645588 & coding \\
\hline Gm10536 & 0.0343469 & noncoding \\
\hline Gm12022-201 & 0.0201895 & noncoding \\
\hline Gm12440 & 0.0262182 & noncoding \\
\hline Gm12610 & 0.0263595 & noncoding \\
\hline Gm14005_Morrbid & 0.156178 & noncoding \\
\hline Gm14636 & 0.509347 & coding \\
\hline Gm14963 & 0.0284942 & noncoding \\
\hline Gm15850 & 0.0998467 & noncoding \\
\hline Gm16062 & 0.586844 & coding \\
\hline Gm16159 & 0.0252371 & noncoding \\
\hline Gm20324 & 0.654366 & coding \\
\hline Gm20753 & 0.195639 & noncoding \\
\hline Gm30505 & 0.103945 & noncoding \\
\hline Gm39090 & 0.088985 & noncoding \\
\hline Gm4827 & 0.354224 & noncoding \\
\hline Gm50039 & 0.776921 & coding \\
\hline Kcnq1ot1 & 0.141033 & noncoding \\
\hline
\end{tabular}


bioRxiv preprint doi: https://doi.org/10.1101/2021.07.14.452339; this version posted July 14,2021 . The copyright holder for this preprint (which was not certified by peer review) is the author/funder, who has granted bioRxiv a license to display the preprint in perpetuity. It is made available under aCC-BY-NC-ND 4.0 International license.

Meg3
Mir100hg
Mir22hg
Neat1
Pdxk-ps-203
Rnf38-217
Slco2a1-202
Slfn10-ps-201
Snhg4
Sox2ot
Speer4cos-201
Speer5-ps1-202
Speer6-ps1-203
Tmcc3-206
Xist_202
9230102004Rik-202
9430078K24Rik

0.408619

0.0765838

noncoding

0.0323014

noncoding

0.178003

noncoding

0.0433202

noncoding

0.0000141858

noncoding

0.0717885

noncoding

0.093136

noncoding

0.0246947

noncoding

0.02483

noncoding

0.065561

noncoding

0.0124209

noncoding

0.0280487

noncoding

0.0109869

noncoding

0.0241659

noncoding

0.0244906

noncoding

0.106114

noncoding

noncoding 